Research Square
Preprints are preliminary reports that have not undergone peer review.

They should not be considered conclusive, used to inform clinical practice, or referenced by the media as validated information.

\title{
Isolated Metastasis of the Ascending Ramus of the Mandible of Thyroid Follicular Carcinoma: A Case Report and Literature Review
}

\author{
Siyao Zhang \\ China-Japan Union Hospital of Jilin University \\ Qingjia Sun \\ China-Japan Union Hospital of Jilin University \\ Dongdong Zhu ( $\nabla$ zhudd@jlu.edu.cn ) \\ China-Japan Union Hospital of Jilin University
}

Case report

Keywords: Follicular thyroid carcinoma, Head and neck surgery, Thyroid neoplasm, Invasion, Bone metastases

Posted Date: October 11th, 2021

DOI: https://doi.org/10.21203/rs.3.rs-903376/v1

License: (c) (7) This work is licensed under a Creative Commons Attribution 4.0 International License. Read Full License 


\section{Abstract}

Objectives: The mandibular metastatic spread of carcinoma from the thyroid gland is exceedingly rare. In August 2020, we treated a 69-year-old woman exhibiting thyroid follicular carcinoma metastasis to the ascending ramus region of the mandible showing evidence of detailed radiological and pathological features.

Methods: We present a case report of thyroid carcinoma that metastasized to the ascending ramus region of the mandible at 21 years after partial thyroidectomy. We also present relevant information in a literature review of 35 articles, consisting of 43 cases (including our case) of thyroid carcinomas with jaw bone metastasis. We statistically analyzed the demographical and clinical results in terms of age, sex, type of primary cancer, site and time of metastasis, treatment, and outcome.

Results: Unlike most of the other cases, in our case, the thyroid cancer had metastasized to the ascending ramus region of the mandible, which made the diagnosis relatively difficult. The patient underwent partial mandibular resection, thyroidectomy, and iodine-131 treatment. The patient was followed up regularly, and no new symptoms were observed at seven months after post-treatment.

Conclusions: Surgery is the most common treatment for thyroid metastases of the mandible. Clinicians should ask thyroid cancer patients for follow-up to monitor whether any new complications have occurred. Patients must undergo complete examination of the maxillofacial bone for up to 40 years or more. Otolaryngologists and stomatologists should pay extra attention to patients with thyroid cancer or nodules to avoid misdiagnosis or missing the recognition of thyroid metastatic cancer.

\section{Background}

Follicular thyroid carcinoma (FTC) is the second most common type of thyroid carcinoma and accounts for approximately $10-15 \%{ }^{[1]}$ of all thyroid cancers. FTC is a slow growing, solitary thyroid nodule ${ }^{[2]}$ that occurs in $0.22-1.35 \%$ of children and $4 \%$ of adults ${ }^{[3]}$. The mean age at onset of FTC is between $40-60$ years, and women are typically more affected than men, with a 3:1 ratio ${ }^{[1]}$. The most common initial treatment for FTC is surgery; however, with disease progression, other therapeutic strategies are employed, including non-radioiodine avid and external beam radiotherapy (EBRT) as well as systemic therapies for unresectable lesions. Although FTC grows inert, its prognosis is not satisfactory. Several factors have been identified in contributing to the poor outcome of FTC. Patients of old age exhibit a propensity for angiovascular invasion of structures, regional lymph nodes, and distant regions, resulting in a high incidence of distant metastasis and mortality ${ }^{[3]}$. The prognosis of FTC is still not satisfactory due to distant metastasis.

Distant metastases develop in $6-20 \%$ of FTC cases and $10-40 \%$ of these cases decrease the 10 -year survival compared with those without distant metastases ${ }^{[4-7]}$. Distant metastases may be the initial presentation of the disease condition or may occur after initial treatment for cancer. ${ }^{[8]}$ The most common organ sites of distant metastasis in FTC are the bones, lungs, and liver. ${ }^{[3]}$ One study found that patients with skeletal metastases did not survive more than 10 years $^{[9]}$. At present, there is no definite treatment strategy for bone metasis (BM) of FTC, especially for skeletal metastases, which may attribute to the loss of large sample data and insufficient research attention. Surgical excision, EBRT, stereotactic body radiation therapy, or other local therapies are

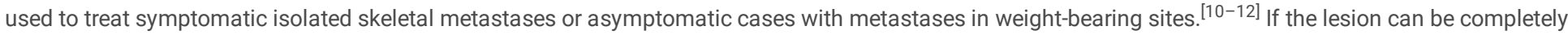
removed, surgical resection is the primary choice ${ }^{[10]}$. Besides, for those who cannot be resected surgically, non-radioactive iodine deficiency and progressive disease, EBRT and systemic therapies can be considered ${ }^{[10]}$

FTC metastasis to the mandible is exceedingly rare and its diagnosis and treatment are challenging. In this case report, a woman possessing FTC metastasizing to the ascending ramus region of the mandible presented a painless lump in the right parotid region. This case was the first case of thyroid cancer with isolated metastasis to the ascending ramus region of the mandible reported in the last three decades. Because of the special anatomical location of this metastasis (adjacent to the temporomandibular joint), which can be misdiagnosed as a primary tumor of the mandible or a parotid gland mass, diagnosis and treatment may be difficult. This case report describes FTC metastatic to the ascending ramus region of the mandible with detailed radiological and pathological features, which may provide insights to help guide future cases.

\section{Methods}

In August 2020, a 69-year-old woman was referred to the otorhinolaryngology department of the China-Japan Union Hospital of Jilin University complaining of facial swelling in the right parotid region without any pain for 2 years. The patient was advised to undergo magnetic resonance imaging (MRI) scan of the head, which confirmed a lump in the right parotid region. She visited our clinic seeking the best treatment options for relief from swelling. During this period, the volume of the mass increased slightly. After examining the case history, we found that she had a long-term history of partial thyroidectomy from an enlarged thyroid gland that caused shortness of breath in 1999. She underwent operation and postoperative pathology reports revealed a right FTC along with capsular and vascular invasion. However, she did not receive any adjuvant treatment after resection. She had no history of tobacco or alcohol abuse, and her body mass index (BMI) was also stable (normal BMI ranges are typically 18.5-24 in China). Further clinical examination detected the existence of a smooth, soft lesion approximately $3 \mathrm{~cm}$ in size in the right parotid region. These complications did not cause any cervical lymphadenopathy and limited mouth opening. Ears, nose, and throat showed normal functions, and facial nerves were intact. Computed tomography (CT) scan was performed on the chest and the results were normal. Therefore, we made a preliminary diagnosis of parotid gland mass and believed that it was a benign mass.

We next performed CT angiography of the carotid, which identified the space-occupying lesions at the right mandible and the lateral pterygoid muscle (Figure 1). This led us to reconsider our initial diagnosis. Even with the ultrasound scan of the cervical lymph nodes, no abnormalities were found. We considered that the mass might be a malignant tumor of the primary mandible and conducted further needle biopsy. Ultrasound-guided needle biopsy of the lump confirmed 
the presence of thyroid follicular cells. Immunohistochemical analysis further confirmed these findings and revealed thyroid transcription factor-1 (TTF-1), paired box gene-8 (PAX-8), galectin-3 and thyroglobulin positivity, as well as partial cytokeratin-7 (CK7) positivity; the expressions of cytokeratin-19 (CK19) and cluster differentiation-56 (CD56) were negative; and the Ki67 index was approximately 5\% (Figure 2). Positron emission tomography-CT was performed; however, the scan reports did not reveal further pathology. Laboratory test results revealed normal T4 and T3 levels, along with a thyroid-stimulating hormone (TSH) level of $4.64 \mathrm{mIU} / \mathrm{L}$ (normal $<4.94 \mathrm{mIU} / \mathrm{L}$ ); however, serum thyroglobulin level was elevated at $108.50 \mathrm{ng} / \mathrm{ml}$ (normal $<23 \mathrm{ng} / \mathrm{ml}$ ).

The patient then underwent a right segmental mandibulectomy. After administration of general anesthesia, an S-shaped incision of $2 \mathrm{~cm}$ was made from the front region of the tragus under the mandible. Intraoperative findings revealed a round mass measuring up to $5 \mathrm{~cm}$ with clear demarcation. The mass was found on the deep face of the facial nerve that enters into the parotid gland of the posteromedial surface reaching the skull base and cervical transverse process and covering the ascending ramus region of the mandible. The facial nerve was free on the surface of the tumor and it was gently pulled upwards to fully expose the superficial surface of the tumor. To release the tumor, the ascending ramus region of the mandible was sawed laterally at $2 \mathrm{~cm}$ below the lower pole of the tumor, and the internal pterygoid muscle was cut off. The external carotid artery was lying adjacent to the tumor, and a branch entered the tumor. The tumor was completely removed after ligation of the external carotid artery (Fig. 3). We performed quick pathological examination of the incisional margin during the operation and found no infiltration of cancer cells at the incisional margin. Immunohistochemical analysis of the tumor indicated TTF- 1 and thyroglobulin positivity and partial CK19 positivity; staining for P53 and CD56 were negative; and the Ki67 index was approximately $5 \%$ (Fig. 4 and Table 1). The integrity of the facial nerve was maintained during the whole operation and there was no facial paralysis during the first 2 days after the surgery. The incisions healed well with little scar formation after 7 days of surgery. Because of the compressive bandage, there was slight swelling and bruising on the right side of the face, and mouth opening was not restricted. After the patient recovered, she was transferred to the thyroid department to undergo total thyroidectomy and postoperative adjuvant therapy, such as radioactive iodine therapy. The patient was followed up regularly, and no new symptoms appeared at seven months of post-treatment.

\section{Results}

We performed a systematic literature review using PubMed and Medline databases for better understanding of the disease. Relevant articles were gathered using the key terms "thyroid," AND "metastasis, "mandible, AND "cancer", "Oral cavity, AND "Orbit". We obtained information on 43 cases (including our case). Among the 43 cases, 35 cases of thyroid carcinoma with jawbone metastasis were identified (Table 2) using appropriate key terms. Among the overall 43 cases, there were 34 female patients, 8 male patients, and 1 patient of unspecified sex. The female predominance, with a male-to-female ratio of 4:17, may be attributed to the higher prevalence of the disease in women ${ }^{[1]}$.

Table 1

Immunohistochemistry results of needle biopsy and postoperative sample

\begin{tabular}{|c|c|c|c|c|c|c|c|c|c|}
\hline & TTF-1 & PAX-8 & galectin-3 & thyroglobulin & CK7 & CK19 & CD56 & Ki67 & P53 \\
\hline Needle biopsy & $t^{*}$ & + & + & + & Scattered(+) & $-\dagger$ & - & $5 \%+$ & $\mathrm{ND}^{\ddagger}$ \\
\hline Postoperative & + & ND & ND & + & ND & Focal weakly(-) & Part(-) & $5 \%+$ & - \\
\hline $\begin{array}{l}\text { * positivity } \\
\text { † negative } \\
\text { ₹ Not described (ND) }\end{array}$ & & & & & & & & & \\
\hline
\end{tabular}


Table 2

Clinical data of 43 cases of thyroid carcinoma with oral metastasis

\begin{tabular}{|c|c|c|c|c|c|c|c|c|}
\hline Author & $\begin{array}{l}\text { Age } \\
\text { (year) }\end{array}$ & Gender & Histology & $\begin{array}{l}\text { Symptom of } \\
\text { metastasis }\end{array}$ & $\begin{array}{l}\text { Time to } \\
\text { metastasis }\end{array}$ & $\begin{array}{l}\text { Location of } \\
\text { metastasis }\end{array}$ & Treatment & $\begin{array}{l}\text { Outcome } \\
\text { (length of } \\
\text { follow-up) }\end{array}$ \\
\hline $\begin{array}{l}\text { Lavanya et al. } \\
\text { [36] }\end{array}$ & 76 & Male & FTC & $\begin{array}{l}\text { Left painless jaw } \\
\text { tumor }\end{array}$ & $\begin{array}{l}\text { First } \\
\text { manifestation }\end{array}$ & $\begin{array}{l}\text { Left mandibular } \\
\text { body }\end{array}$ & $\begin{array}{l}\text { Refer to an } \\
\text { oncologist, not report }\end{array}$ & Not report \\
\hline \multirow{4}{*}{$\begin{array}{l}\text { Kundan } \\
\text { Kisanrao Nawale } \\
\text { et al. }{ }^{[3]}\end{array}$} & 60 & Female & PTC & Not report & $\begin{array}{l}\text { No (not } \\
\text { report) }\end{array}$ & Mandible & Mandibulectomy & Not report \\
\hline & 42 & Female & PTC & Not report & $\begin{array}{l}\text { No (not } \\
\text { report) }\end{array}$ & Mandible & Biopsy, not report & Not report \\
\hline & 50 & Female & PTC & Not report & $\begin{array}{l}\text { No (not } \\
\text { report) }\end{array}$ & Mandible & Mandibulectomy & Not report \\
\hline & 56 & Female & PTC & Not report & $\begin{array}{l}\text { No (not } \\
\text { report) }\end{array}$ & Mandible & Mandibulectomy & Not report \\
\hline $\begin{array}{l}\text { Ihsen Slim et al. } \\
\text { [38] }\end{array}$ & 67 & Female & PTC & $\begin{array}{l}\text { Left pain cheek } \\
\text { tumor }\end{array}$ & 7years & $\begin{array}{l}\text { Left malar, } \\
\text { maxilla and } \\
\text { orbit }\end{array}$ & $\begin{array}{l}\text { Surgical resection of } \\
\text { the malar mass, } \\
\text { thyroidectomy, } \\
\text { radioiodine (I131) } \\
\text { therapy }\end{array}$ & $\begin{array}{l}\text { Alive (not } \\
\text { report) }\end{array}$ \\
\hline $\begin{array}{l}\text { Algahtani et al. } \\
\text { [39] }\end{array}$ & 66 & Female & FTC & $\begin{array}{l}\text { Left cheek } \\
\text { swelling, lower lip } \\
\text { numbness, } \\
\text { difficulty chewing, } \\
\text { pathological } \\
\text { fracture }\end{array}$ & $\begin{array}{l}\text { First } \\
\text { manifestation }\end{array}$ & $\begin{array}{l}\text { Left mandibular } \\
\text { body and ramus }\end{array}$ & $\begin{array}{l}\text { Total thyroidectomy, } \\
\text { lateral neck } \\
\text { dissection, } \\
\text { mandibular lesion } \\
\text { resection }\end{array}$ & $\begin{array}{l}\text { Alive (not } \\
\text { report) }\end{array}$ \\
\hline $\begin{array}{l}\text { Nikolaos Get } \\
\text { al }^{[40]}\end{array}$ & 63 & Male & PTC & $\begin{array}{l}\text { Right painful } \\
\text { maxillary swelling }\end{array}$ & 2 years & $\begin{array}{l}\text { Right maxilla, } \\
\text { sternum, ribs, } \\
\text { left tibia }\end{array}$ & $\begin{array}{l}\text { Radiotherapy, } \\
\text { chemotherapy }\end{array}$ & Alive (2 years) \\
\hline $\begin{array}{l}\text { A Agarwal et al, } \\
\text { [41] }\end{array}$ & 45 & Female & FTC & Facial swelling & $\begin{array}{l}\text { First } \\
\text { manifestation }\end{array}$ & $\begin{array}{l}\text { Left mandibular } \\
\text { body }\end{array}$ & Hemimandibulectomy & $\begin{array}{l}\text { Alive (2 } \\
\text { weeks) }\end{array}$ \\
\hline S Anil et al. ${ }^{[19]}$ & 61 & Female & FTC & $\begin{array}{l}\text { Right pain } \\
\text { swelling of the } \\
\text { mandibular angle, } \\
\text { erythematous } \\
\text { change in oral } \\
\text { mucosa }\end{array}$ & 8 years & $\begin{array}{l}\text { Right } \\
\text { mandibular } \\
\text { angle and } \\
\text { ramus }\end{array}$ & $\begin{array}{l}\text { Incision biopsy, not } \\
\text { report }\end{array}$ & Not report \\
\hline $\begin{array}{l}\text { Chetan J } \\
\text { Bhadage et al, }{ }^{[42]}\end{array}$ & 40 & Female & FTC & $\begin{array}{l}\text { Lower left facial } \\
\text { swelling, neck } \\
\text { lump, tooth } \\
\text { extraction }\end{array}$ & $\begin{array}{l}\text { First } \\
\text { manifestation }\end{array}$ & $\begin{array}{l}\text { Left mandibular } \\
\text { body }\end{array}$ & $\begin{array}{l}\text { Biopsy, transfer } \\
\text { treatment (not report) }\end{array}$ & Not report \\
\hline $\begin{array}{l}\text { Caliaperoumal } \\
\text { Santhosh Kumar } \\
\text { et al, }{ }^{[43]}\end{array}$ & 31 & Female & FTC & $\begin{array}{l}\text { Painful swelling, } \\
\text { mobile teeth }\end{array}$ & $\begin{array}{l}\text { First } \\
\text { manifestation }\end{array}$ & $\begin{array}{l}\text { Posterior } \\
\text { maxilla }\end{array}$ & $\begin{array}{l}\text { Thyroidectomy, } \\
\text { radioiodine (I131) } \\
\text { therapy }\end{array}$ & Alive (7years) \\
\hline T Heferet al, ${ }^{[44]}$ & 52 & Male & FTC & $\begin{array}{l}\text { Left hard palate } \\
\text { discomfort }\end{array}$ & Six months & Left maxilla & Left maxillectomy & Alive (2 years) \\
\hline $\begin{array}{l}\text { Mahnaz } \\
\text { Fatahzadehet al. } \\
\text { [45] }\end{array}$ & 43 & Female & PTC & $\begin{array}{l}\text { Hemorrhagicmass } \\
\text { in the right maxilla } \\
\text { noted }\end{array}$ & $\begin{array}{l}\text { No (not } \\
\text { report) }\end{array}$ & Right maxilla & Radiotherapy & Not report \\
\hline \multirow{3}{*}{$\begin{array}{l}\text { Antonio Azoubel } \\
\text { Antunes et al. }{ }^{[46]}\end{array}$} & 13 & Female & PTC & Not report & Not report & Maxilla & Not report & Not report \\
\hline & 64 & Female & FTC & Not report & Not report & Mandible & Not report & Not report \\
\hline & 75 & Female & FTC & Not report & Not report & Mandible & Not report & Not report \\
\hline 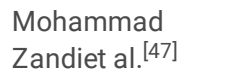 & 73 & Male & $\mathrm{TC}$ & $\begin{array}{l}\text { Right painful } \\
\text { mandible mass }\end{array}$ & $\begin{array}{l}\text { First } \\
\text { manifestation }\end{array}$ & $\begin{array}{l}\text { Body of right } \\
\text { mandible }\end{array}$ & Palliative therapy & Not report \\
\hline $\begin{array}{l}\text { Ugur Anil Bingol } \\
\text { et al. }{ }^{[48]}\end{array}$ & 33 & Female & PTC & $\begin{array}{l}\text { Right angulus } \\
\text { mandibular mass }\end{array}$ & 13 years $^{*}$ & $\begin{array}{l}\text { Right angulus } \\
\text { mandible }\end{array}$ & $\begin{array}{l}\text { Right } \\
\text { hemimandibulectomy } \\
\text { and reconstructed } \\
\text { with costochondral } \\
\text { rib graft }\end{array}$ & $\begin{array}{l}\text { Death due to } \\
\text { multisystem } \\
\text { metastases ( } 5 \\
\text { years) }\end{array}$ \\
\hline $\begin{array}{l}\text { Giuseppe Colella } \\
\text { et al. }{ }^{[49]}\end{array}$ & 50 & Female & PTC & $\begin{array}{l}\text { Pain and swelling } \\
\text { in right mandible }\end{array}$ & 5 years & $\begin{array}{l}\text { Right } \\
\text { mandibular } \\
\text { body }\end{array}$ & Not report & Not report \\
\hline $\begin{array}{l}\text { B W Draper et al. } \\
\text { [50] }\end{array}$ & $\begin{array}{l}\text { Not } \\
\text { report }\end{array}$ & Female & PTC & Oral ulcers & Not report & $\begin{array}{l}\text { Mandibularbody, } \\
\text { skull }\end{array}$ & Radiotherapy & Not report \\
\hline
\end{tabular}




\begin{tabular}{|c|c|c|c|c|c|c|c|c|}
\hline Author & $\begin{array}{l}\text { Age } \\
\text { (year) }\end{array}$ & Gender & Histology & $\begin{array}{l}\text { Symptom of } \\
\text { metastasis }\end{array}$ & $\begin{array}{l}\text { Time to } \\
\text { metastasis }\end{array}$ & $\begin{array}{l}\text { Location of } \\
\text { metastasis }\end{array}$ & Treatment & $\begin{array}{l}\text { Outcome } \\
\text { (length of } \\
\text { follow-up) }\end{array}$ \\
\hline T Erdaget al. ${ }^{[51]}$ & 53 & Female & PTC & $\begin{array}{l}\text { Right cheek } \\
\text { swelling }\end{array}$ & Not report & $\begin{array}{l}\text { Right mandible } \\
\text { (not report) }\end{array}$ & Surgery, radiotherapy & $\begin{array}{l}\text { Alive }(2.5 \\
\text { years) }\end{array}$ \\
\hline $\begin{array}{l}\text { L Essakalli et al. } \\
\text { [52] }\end{array}$ & 50 & Female & PTC & $\begin{array}{l}\text { Painful jaw } \\
\text { swelling }\end{array}$ & Not report & $\begin{array}{l}\text { Mandible (not } \\
\text { report) }\end{array}$ & Surgery, radiotherapy & $\begin{array}{l}\text { Alive (2 } \\
\text { months) }\end{array}$ \\
\hline $\begin{array}{l}\text { M A Germain et } \\
\text { al. }{ }^{[53]}\end{array}$ & 50 & Female & PTC & Lymphadenopathy & 13 years & $\begin{array}{l}\text { Mandibular } \\
\text { body }\end{array}$ & $\begin{array}{l}\text { Interruptive } \\
\text { mandibulectomy, } \\
\text { reconstruction with a } \\
\text { free composed } \\
\text { vascularized fibular } \\
\text { transplant }\end{array}$ & $\begin{array}{l}\text { Alive (17 } \\
\text { months) }\end{array}$ \\
\hline $\begin{array}{l}\text { Sumairi Bin } \\
\text { Ismailet al. }^{[17]}\end{array}$ & 70 & Female & FTC & $\begin{array}{l}\text { Painful swelling in } \\
\text { the left side of the } \\
\text { angle of the } \\
\text { mandible, loose } \\
\text { teeth }\end{array}$ & $\begin{array}{l}\text { First } \\
\text { manifestation }\end{array}$ & $\begin{array}{l}\text { Left mandibular } \\
\text { body }\end{array}$ & Biopsy, not report & Not report \\
\hline $\begin{array}{l}\text { M A Kahn et al. } \\
\text { [34] }\end{array}$ & 82 & Female & FTC & $\begin{array}{l}\text { Painful } \\
\text { mandibular } \\
\text { vestibular swelling }\end{array}$ & 32 years $^{\dagger}$ & Mandibularbody & $\begin{array}{l}\text { Radiotherapy, } \\
\text { radioactive } \\
\text { iodine therapy, } \\
\text { resection }\end{array}$ & $\begin{array}{l}\text { Died from } \\
\text { cardiovascular } \\
\text { failure ( } 1.5 \\
\text { years) }\end{array}$ \\
\hline $\begin{array}{l}\text { Rayadurgam } \\
\text { Venkata Kishore } \\
\text { Kumar et al. }{ }^{[54]}\end{array}$ & 58 & Female & FTC & $\begin{array}{l}\text { Slow-growing } \\
\text { painless swelling } \\
\text { on the left lower } \\
\text { jaw }\end{array}$ & $\begin{array}{l}\text { First } \\
\text { manifestation }\end{array}$ & $\begin{array}{l}\text { Left body of the } \\
\text { mandible }\end{array}$ & $\begin{array}{l}\text { Segmental } \\
\text { mandibular resection, } \\
\text { total thyroidectomy } \\
\text { with midline neck } \\
\text { dissection, } \\
\text { postoperative } \\
\text { radioiodine therapy }\end{array}$ & Alive (2 years) \\
\hline E Vuralet al. ${ }^{[20]}$ & 64 & Female & FTC & $\begin{array}{l}\text { Right } \\
\text { progressively } \\
\text { enlarging } \\
\text { preauricular mass }\end{array}$ & $\begin{array}{l}\text { First } \\
\text { manifestation }\end{array}$ & $\begin{array}{l}\text { Right } \\
\text { mandibular } \\
\text { angle and } \\
\text { ramus }\end{array}$ & $\begin{array}{l}\text { Totalthyroidectomy, } \\
\text { radical resection, } \\
\text { radioactive } \\
\text { iodine therapy }\end{array}$ & Not report \\
\hline $\begin{array}{l}\text { Paranjyothi } \\
\text { Magadi } \\
\text { Vishveshwaraiah } \\
\text { et al. }^{[55]}\end{array}$ & 56 & Female & FTC & $\begin{array}{l}\text { Right painless } \\
\text { lower facial } \\
\text { swelling, } \\
\text { paresthesia }\end{array}$ & $\begin{array}{l}\text { First } \\
\text { manifestation }\end{array}$ & $\begin{array}{l}\text { Right } \\
\text { mandibular } \\
\text { body }\end{array}$ & $\begin{array}{l}\text { Biopsy, referred to an } \\
\text { oncologist }^{\ddagger}\end{array}$ & Not report \\
\hline $\begin{array}{l}\text { Leila Vazifeh } \\
\text { Mostaan et al. }{ }^{[56]}\end{array}$ & 58 & Female & FTC & $\begin{array}{l}\text { Right facial } \\
\text { swelling on } \\
\text { mandibular angle, } \\
\text { teeth extraction }\end{array}$ & 12 years & $\begin{array}{l}\text { Right } \\
\text { mandibular } \\
\text { angle and } \\
\text { ramus }\end{array}$ & $\begin{array}{l}\text { Segmental } \\
\text { mandibulectomy }\end{array}$ & Not report \\
\hline F Tovi et al. ${ }^{[57]}$ & 33 & Male & FTC & $\begin{array}{l}\text { Large intraoral } \\
\text { mass of the left } \\
\text { molar area }\end{array}$ & 10 years & $\begin{array}{l}\text { Left mandibular } \\
\text { angle and } \\
\text { ramus }\end{array}$ & $\begin{array}{l}\text { Radioactive iodine } \\
\text { therapy§ }\end{array}$ & $\begin{array}{l}\text { Died of thyroid } \\
\text { crisis ( } 17 \\
\text { days) }\end{array}$ \\
\hline \multirow[t]{3}{*}{$\begin{array}{l}\text { Demetrio } \\
\text { Tamiolakis et al. } \\
\text { [18] }\end{array}$} & 69 & Female & PTC & $\begin{array}{l}\text { Facial swelling, } \\
\text { nasal obstruction, } \\
\text { epistaxis }\end{array}$ & Not report & $\begin{array}{l}\text { Mandible (not } \\
\text { report) }\end{array}$ & Inoperable & $\begin{array}{l}\text { Died of } \\
\text { disease(1 } \\
\text { month) }\end{array}$ \\
\hline & $\begin{array}{l}\text { Not } \\
\text { report }\end{array}$ & $\begin{array}{l}\text { Not } \\
\text { report }\end{array}$ & TC & $\begin{array}{l}\text { Facial swelling, } \\
\text { oral }\end{array}$ & Not report & $\begin{array}{l}\text { Mandible (not } \\
\text { report) }\end{array}$ & Resection & Not report \\
\hline & & & & $\begin{array}{l}\text { mucosal } \\
\text { ulcerations }\end{array}$ & & & & \\
\hline $\begin{array}{l}\text { Ajay Prakash } \\
\text { Pasupula et al. } \\
\text { [58] }\end{array}$ & 40 & Female & FTC & $\begin{array}{l}\text { Painful swelling in } \\
\text { the left parotid } \\
\text { region, intermittent } \\
\text { low-grade fever }\end{array}$ & $\begin{array}{l}\text { First } \\
\text { manifestation }\end{array}$ & $\begin{array}{l}\text { Left mandibular } \\
\text { body and ramus }\end{array}$ & $\begin{array}{l}\text { Total thyroidectomy, } \\
\text { resection of } \\
\text { metastatic tumor of } \\
\text { the mandible }\end{array}$ & Not report \\
\hline $\begin{array}{l}\text { Alejandro } \\
\text { Ostrosky et al. }{ }^{[59]}\end{array}$ & 72 & Male & FTC & Slight toothache & $\begin{array}{l}\text { First } \\
\text { manifestation }\end{array}$ & $\begin{array}{l}\text { Left mandibular } \\
\text { body }\end{array}$ & $\begin{array}{l}\text { Total thyroidectomy, } \\
\text { resection and } \\
\text { reconstruction of the } \\
\text { mandible }\end{array}$ & Not report \\
\hline I Meyeret al. ${ }^{[60]}$ & 51 & Female & FTC & Not report & Not report & Not report & Not report & Not report \\
\hline Lin Liu et al. ${ }^{[35]}$ & 66 & Male & FTC & Left cheek lump & 22 years & $\begin{array}{l}\text { Mandible (not } \\
\text { report) }\end{array}$ & $\begin{array}{l}\text { Radical resection of } \\
\text { the mandibular tumor, } \\
\text { radioactive iodine } \\
\text { therapy }\end{array}$ & Alive (3years) \\
\hline
\end{tabular}




\begin{tabular}{|c|c|c|c|c|c|c|c|c|}
\hline Author & $\begin{array}{l}\text { Age } \\
\text { (year) }\end{array}$ & Gender & Histology & $\begin{array}{l}\text { Symptom of } \\
\text { metastasis }\end{array}$ & $\begin{array}{l}\text { Time to } \\
\text { metastasis }\end{array}$ & $\begin{array}{l}\text { Location of } \\
\text { metastasis }\end{array}$ & Treatment & $\begin{array}{l}\text { Outcome } \\
\text { (length of } \\
\text { follow-up) }\end{array}$ \\
\hline $\begin{array}{l}\text { JD Osguthorpeet } \\
\text { al. }^{[28]}\end{array}$ & 53 & Male & FTC & $\begin{array}{l}\text { Slowly enlarging } \\
\text { right parotid area } \\
\text { mass }\end{array}$ & $\begin{array}{l}\text { First } \\
\text { manifestation }\end{array}$ & $\begin{array}{l}\text { Right } \\
\text { mandibular } \\
\text { ramus }\end{array}$ & $\begin{array}{l}\text { Partial } \\
\text { mandibulectomy }\end{array}$ & Alive (3 years) \\
\hline $\begin{array}{l}\text { H Nishikawa et } \\
\text { al. }^{[61]}\end{array}$ & 83 & Female & FTC & $\begin{array}{l}\text { Swelling and pain } \\
\text { in the median } \\
\text { lower jaw }\end{array}$ & 2 years & $\begin{array}{l}\text { Median } \\
\text { mandible }\end{array}$ & None & $\begin{array}{l}\text { Died of } \\
\text { disease(19 } \\
\text { months) }\end{array}$ \\
\hline $\begin{array}{l}\text { A Markitziu et al. } \\
\text { [29] }\end{array}$ & 69 & Female & PTC & $\begin{array}{l}\text { Left facial } \\
\text { swelling }\end{array}$ & $\begin{array}{l}\text { First } \\
\text { manifestation }\end{array}$ & $\begin{array}{l}\text { Left ascending } \\
\text { ramus of the } \\
\text { mandible and } \\
\text { parotid }\end{array}$ & $\begin{array}{l}\text { Irradiation, } \\
\text { chemotherapy }\end{array}$ & $\begin{array}{l}\text { Alive }(1.5 \\
\text { years) }\end{array}$ \\
\hline \multirow[t]{2}{*}{$\begin{array}{l}\text { R K McDaniel et } \\
\text { al. }{ }^{[62]}\end{array}$} & 77 & Female & FTC & $\begin{array}{l}\text { Pain and swelling } \\
\text { in the right } \\
\text { mandible }\end{array}$ & $\begin{array}{l}\text { First } \\
\text { manifestation }\end{array}$ & $\begin{array}{l}\text { Right } \\
\text { mandibular } \\
\text { posterior body } \\
\text { and ramus }\end{array}$ & $\begin{array}{l}\text { Right } \\
\text { hemimandibulectomy, } \\
\text { total parotidectomy }\end{array}$ & Alive (4 years) \\
\hline & 48 & Female & FTC & $\begin{array}{l}\text { Swelling in left } \\
\text { mandible of } \\
\text { thyroid parotid } \\
\text { area, numbness of } \\
\text { left lower lip }\end{array}$ & $\begin{array}{l}\text { First } \\
\text { manifestation }\end{array}$ & $\begin{array}{l}\text { Left mandibular } \\
\text { ramus }\end{array}$ & Not report & $\begin{array}{l}\text { Alive (not } \\
\text { report) }\end{array}$ \\
\hline Present case & 69 & Female & FTC & $\begin{array}{l}\text { Painless lump in } \\
\text { the right parotid } \\
\text { region }\end{array}$ & 21 years & $\begin{array}{l}\text { Right ascending } \\
\text { ramus of the } \\
\text { mandible }\end{array}$ & $\begin{array}{l}\text { Right segmental } \\
\text { mandibulectomy, } \\
\text { thyreoidectomy, } \\
\text { radioactive iodine } \\
\text { therapy }\end{array}$ & $\begin{array}{l}\text { Alive }(7 \\
\text { months })\end{array}$ \\
\hline
\end{tabular}

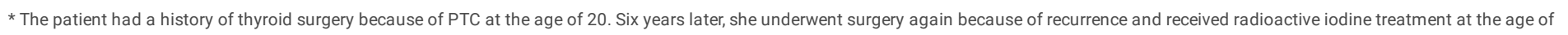
30. The patient underwent two surgeries due to metastases of the pelvis and humerus

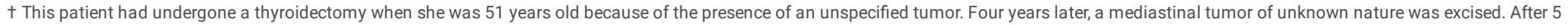

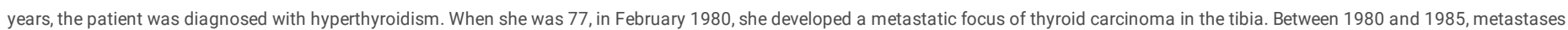
invaded the lungs and pelvis.

‡ The patient had undergone an incisional biopsy elsewhere, which was diagnosed as an odontogenic tumor

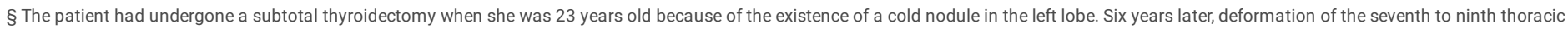

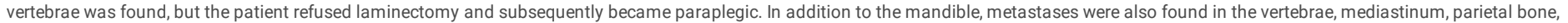
and femur

The patient age ranged from 13 years to 83 years, and the median age of patients was 57 years. This observation is consistent with the fact that metastatic tumors are reported to be the most common diseases in the fifth to seventh decade of life ${ }^{[13]}$. Among the histological types of thyroid carcinoma, 25 of the 43 cases $(58.1 \%)$ were follicular carcinomas and 16 of these cases (37\%) were considered as papillary carcinoma; for the remaining 2 cases, the primary tumor was reported as thyroid carcinoma without further clinical description. Although the incidence of papillary thyroid carcinoma (PTC) was higher than FTC, FTC shows high vascularity and a higher rate of vascular invasion ${ }^{[14]}$. This may account for the higher incidence of FTC with maxillofacial metastasis.

The clinical symptoms of maxillofacial metastasis include pain in the gums, swelling, loosening of tooth, paraesthesia of the lower lip and chin, trismus, growth of tumor projecting out from the soft tissue, and pathologic fracture ${ }^{[15]}$. The lesion can also cause pain in the temporomandibular joint region or as osteomyelitis in the jaw or as trigeminal neuralgia ${ }^{[16]}$. However, some cases do not show symptoms.Sometimesthe symptoms or signs may be the only manifestation of an undiscovered malignancy ${ }^{[17]}$. Among the 43 cases, 16 cases were diagnosed with distant metastasis as the initial presentation, while in 9 cases, the timing between the diagnosis of the primary and metastatic disease was not specified. In the other 18 cases, patients developed distant metastases after the initial treatment for thyroid carcinoma, with an average of 7 years after treatment (ranging from 6 months to 32 years). This may be related to the slow growth rate of thyroid carcinoma and thus metastases in the bone may be undetected or may remain dormant for a longer period.

Oral metastatic carcinoma accounts for approximately $1 \%$ of all oral cancers ${ }^{[18]}$. Compared with metastases in other bones, metastatic tumors to the jawbones are very rare and the true incidence is not known. The most frequent location for metastasis in the jawbone is the mandible. We found that 36 cases out of the 43 identified cases had metastasis in the mandible. Metastasis to the maxilla was seen in 6 cases out of 43 cases. Most (84\%) metastasis involving jaw bones occurs in the mandible, and the molar area is the most frequent site of metastasis. ${ }^{[18,19]}$ This may be due to the presence of more hematopoietic tissue in the mandible ${ }^{[20]}$. Patients with metastases in the maxilla were relatively young, with an average age of 45 years; in comparison, the mean age of patients with metastasis to the mandible was 59 years.

Rection, EBRT, or embolization for bone metastases in patients in whom these are symptomatic ${ }^{[21]}$. Unfortunately, only a few cases have been cured after the diagnosis of bone metastases with FTC. Recent studies have documented the significant role of the sodium iodide symporter (NIS) in several extrathyroidal cancers such as pancreatic cancer, prostate cancer, colon cancer, ovarian cancer, breast cancer, cervical cancer, and testicular cancer [22] Moreover, balanced regulation of the transcription and translocation of NIS is monitored by $\mathrm{TSH}^{[23]}$. NIS is also essential to establish an $\mathrm{I}^{-}$gradient in thyrocytes, thereby achieving the synthesis of thyroid hormones ${ }^{[24]}$. Furthermore, various studies have reported overexpression of NIS in thyroid cancer cells and its oncogenic 
effects. Hence, the overexpression of NIS in cancer cells has indicated the utility of radioiodide treatment application for the targeting of thyroid cancer [25]. Radioactive iodide (131I) has been used as the standard treatment for FTC with distant metastases of lung or bone, and treatment results are dependent on the expression of NIS expression in cancer cells ${ }^{[25]}$. The efficacy of 131 I treatment for FTC depends on the differentiation of the cancer cells ${ }^{[26]}$. In our literature review, 23 cases underwent surgery and 2 cases (a total of 5 deaths in 43 cases) died. This may also indicate that the prognosis of jawbone metastases is better than that of other bone metastases for thyroid carcinoma (TC). There is evidence that resection of solitary bony metastasis, along with total thyroidectomy, may increase the survival among patients with TC, possibly due to increased radioactive iodine uptake and effects used to treat the potential micrometastases elsewhere in the body after surgery ${ }^{[20]}$. Extremely high vascular density in FTC with bone metastases may not only lead to diagnostic dilemmas but also high surgical mortality and morbidity ${ }^{[20]}$. Therefore, we should focus on the selection of surgical methods and the avoidance of bleeding and post-surgery complications. In recent decades, the extent of surgery for TC has changed. Surgery has become more conservative, with an emphasis on treating the patient on a case-by-case basis. The clinician and patients should discuss the expectations regarding the quality of life as well as the risks and benefits associated with the treatment strategy. This will allow for the best personalized treatment option. Treatment decisions must weigh the rate of tumor progression and quality of life without treatment against the efficacy and side effects of therapy.

\section{Discussion}

Our literature review identified 390 articles that mentioned metastasis in mandibular and maxillary bones. Hirshberg et al ${ }^{[14]}$ observed that breast is the most frequent primary site of metastasis in women while the primary sites of metastatic carcinoma of the mandible region are mainly lung cancer in men [27]. Of the maxillofacial metastases, $60-80 \%$ occur in the mandible and are mainly concentrated in the molar and premolar areas ${ }^{[18]}$. Metastases to the mandible from thyroid cancer are uncommon.

Of the 43 cases we collected, the case reported by Osguthorpe ${ }^{[28]}$ alone has a complaint of parotid sialography, which was diagnosed and a lateral displacement of the superficial lobe and destruction of the mandible from the angle to the neck of the condyle were found. This case occurred in the 1980 s when the technology did not provide any complete images of the mandible. Furthermore, the authors selected the partial mandibulectomy and inferior alveolar nerve en bloc specimen for pathological examination, so whether the tumor is just invading the ascending ramus region of the mandible is not clear. Another case reported by Markitziu ${ }^{[29]}$ involved the ascending ramus region of the mandible and the parotid gland. Although the tumor mass in our case report also consists of the parotid region, which lies between the superficial and deep lobes of the parotid gland, the parotid gland was not invaded and the capsule of the parotid gland was intact. Therefore, our case was the first to report thyroid cancer isolated metastasis to the ascending ramus region of the mandible in the last three decades.

Metastasis is the result of a complex biological cascade that begins when tumor cells detach from the primary tumor and spread into the tissue; the cells then invade lymphatic vascular structures and survive in circulation ${ }^{[13]}$. The microvascular system of the target organ provides a hiding place for metastatic tumor cells, which spread, proliferate and invade the target tissue. Angiogenesis is necessary to provide adequate oxygen and nutrients when tumor cells are overloaded with more than $2-3 \mathrm{~mm}^{[30]}$. Because the mode of metastasis is hematogenous, the neoplastic cells are deposited in areas where there is vascular tissue $^{[31]}$. Some investigators believe that metastasis to the jawbone through the hematogenous route requires the presence of hematopoietically active bone marrow that is well connected with the sinusoidal vascular spaces at the site of deposition of malignant cells ${ }^{\text {[32] }}$. This may be one of the reasons why the involvement of the jaw in metastasis seems to be less common than other bones. The amount of red bone marrow and blood vessels in the jaw tends to decrease with age, which may be from the gradual replacement of red bone marrow by yellow or fatty bone marrow ${ }^{[13]}$. We found that jawbones are not always included in radiographic skeletal surveys for metastasis, according to the National Comprehensive Cancer Network (NCCN) guidelines ${ }^{[10]}$ for thyroid cancer treatment, which does not mention the mandible in the periodic bone examination of patients after thyroid cancer surgery. Therefore, clinicians should also monitor the maxillofacial features in the diagnosis and follow-up of patients with thyroid cancer, rather than focusing only on the common sites of metastasis such as lung and bone.

The most common site of metastasis to the mandible is the molar area ${ }^{[18,19]}$. There are usually no significant blood vessels or nerves in this area, and the main considerations may be the amount of blood loss during the operation and how the mandible is reconstructed. Unlike most previous case reports, in this case, the mass was located close to the temporomandibular joint, which makes the diagnosis difficult. It is difficult to distinguish from the primary mandibular tumor or parotid gland tumor by imaging and clinical manifestations. Therefore, preoperative puncture biopsy is particularly important. A clear diagnosis of the disease is very important to the choice of treatment. Extremely high vascular density in FTC with bone metastases may not only lead to diagnostic dilemmas but also may lead to high surgical mortality and morbidity. Therefore, CT or MRI alone cannot fully evaluate the condition of the tumor; hence, CT angiography or MRI angiography is necessary to further resolve the tumor condition. Vural et al ${ }^{[20]}$ reported a case of FTC metastasis to the mandible with preoperative selective embolization. We evaluated preoperatively and decided not to perform the embolization process. Although we reported the existence of vascular abnormalities of the tumor, in this case, intraoperative blood loss was within a normal range. After the surgery, there was some bleeding due to compression, and the skin of the patient returned to normal within two weeks. We can make a better decision whether to choose preoperative embolization with the help of CT angiography and other imaging techniques. FTC metastatic to mandible can cause significant morphological, functional and esthetic alterations due to disease progression, so it needs aggressive therapy. In terms of reconstruction of the mandible, this case is different from other cases. Instead of using fibula or other bone with blood vessels, our preoperative vision was to use an in vitro titanium plate tailored to the preoperative normal jaw bone of the patient. The patient would be advised to wear it or remove it as needed, just like dentures. However, the patient's occlusal condition and appearance did not appear to be a major problem after the surgery, and the pre-customized titanium plate was not used.

Pruckmayer et al ${ }^{[33]}$ conducted a major retrospective study of 763 patients with nonspecific pain in the mandible, and only 9 patients (1.2\%) had mandibular metastases. They projected the pain as an important marker in the study ${ }^{[3]}$. Most of the cases we collected also reported pain or other symptoms, such as 
lost teeth and numbness of the skin. However, the patient in our case did not experience any discomfort. This may also be a factor in the discovery of metastases after 21 years of partial thyroidectomy. The period of thyroid cancer metastasis may be relatively long. In addition to our case, the period of thyroid cancer metastasis in the case reported by Kahnet al ${ }^{[34]}$ was for 32 years and that in the other case reported by Liu et al ${ }^{[35]}$ was for 22 years. Although this is rare, these facts are also consistent with the indolent process of well-differentiated thyroid carcinoma. This also raises the question of whether the follow-up of thyroid cancer patients should be extended for at least 40 years.

This rare case highlights the importance of a clinical maxillofacial malignancy that is likely to metastasize from other sites and the need for follow-up of patients with malignancy. As thyroidists, we should follow patients with thyroid follicular carcinoma for at least 40 years, and the follow-up should include the maxillofacial area. As otolaryngology and stomatology physicians, it is important to carefully review the patient's history and pay attention to the entire case history of thyroid lumps when diagnosing maxillofacial masses, especially in women. When maxillofacial masses cannot be diagnosed, careful clinical examination combined with radiological, histological, and immunohistochemical assessments can lead to early detection of the primary tumor, more appropriate treatment, and a better prognosis for these patients.

\section{Conclusion}

Because of the rare occurrence of oral and facial metastatic tumors and deceptive clinical and histopathological features, it is often difficult to diagnose this disease. The diagnosis is more challenging when oral and facial metastases are the first manifestations of distant, undetected malignancy. Some highlights of the current case are as follows: (1) this is first reported case of solitary thyroid cancer metastatic to the ascending ramus region of the mandible in nearly 30 years; (2) the period of metastasis was relatively long; (3) the patient reported facial swelling without pain and other special clinical manifestations; and (4) the anatomical location of metastatic carcinoma makes the diagnosis and operation difficult. Although this is a rare occurrence, practitioners should always be highly suspicious when diagnosing patients with oral-facial metastases. The ascending ramus region of the mandible should also be examined in the follow-up period and the time of follow-up should be prolonged.

\section{Abbreviations}

\section{FTC}

Follicular thyroid carcinoma

EBRT

external beam radiotherapy

BM

bone metasis

MRI

magnetic resonance imaging

BMI

body mass index

CT

computed tomography

TTF-1

thyroid transcription factor-1

\section{PAX-8}

paired box gene-8

CK7

cytokeratin-7

CK19

cytokeratin-19

CD56

cluster differentiation-56

TSH

thyroid-stimulating hormone

PTC

papillary thyroid carcinoma

NIS

sodium iodide symporter

TC

thyroid carcinoma

\section{NCCN}

National Comprehensive Cancer Network

ND

Not described

\section{Declarations}


Not applicable.

\section{Funding}

Not applicable.

\section{Author information}

Authors' contributions

All authors have contributed significantly: DZ and SZ designed the study; DZ, SZ, and QS analyzed and interpreted data; DZ, SZ, and QS wrote and/or reviewed the manuscript; all authors discussed the results, commented on the manuscript, and saw and approved this manuscript.

Corresponding author

Correspondence to Dongdong Zhu.

\section{Ethics declarations}

Ethics approval and consent to participate

The case study and treatment plan were approved by the Institutional Review Board and Ethics Committee of The China-Japan Union Hospital of Jilin University.

Consent for publication

Written informed consent was obtained from the patient according to the federal and institutional guidelines.

Competing interests

The authors declare that they have no competing interests.

\section{Availability of data and materials}

Data sharing not applicable to this article as no datasets were generated or analysed during the current study.

\section{References}

1. D'Avanzo A, Treseler P, Ituarte PH, Wong M, Streja L, Greenspan FS, et al. Follicular thyroid carcinoma: histology and prognosis[J]. Cancer. 2004;100(6):1123-9.

2. Mazzaferri EL, Jhiang SM. Long-term impact of initial surgical and medical therapy on papillary and follicular thyroid cancer[J]. Am J Med. 1994;97(5):418-28.

3. Haigh PI. Follicular thyroid carcinoma[J]. Curr Treat Options Oncol. 2002;3(4):349-54.

4. Lin JD, Huang MJ, Juang JH, Chao TC, Huang BY, Chen KW, et al. Factors related to the survival of papillary and follicular thyroid carcinoma patients with distant metastases[J]. Thyroid. 1999;9(12):1227-35.

5. Mihailovic J, Stefanovic L, Malesevic M. Differentiated thyroid carcinoma with distant metastases: probability of survival and its predicting factors[J]. Cancer Biother Radiopharm. 2007;22(2):250-5.

6. Schlumberger M, Challeton C, De Vathaire F, Travagli JP, Gardet P, Lumbroso JD, et al. Radioactive iodine treatment and external radiotherapy for lung and bone metastases from thyroid carcinoma[J]. J Nucl Med. 1996;37(4):598-605.

7. O'Neill CJ, Oucharek J, Learoyd D, Sidhu SB. Standard and emerging therapies for metastatic differentiated thyroid cancer[J]. Oncologist. 2010;15(2):146-56.

8. Shaha AR, Shah JP, Loree TR. Differentiated thyroid cancer presenting initially with distant metastasis[J]. Am J Surg. 1997;174(5):474-6.

9. Brown AP, Greening WP, McCready VR, Shaw HJ, Harmer CL. Radioiodine treatment of metastatic thyroid carcinoma: the Royal Marsden Hospital experience[J]. Br J Radiol. 1984;57(676):323-7.

10. Haddad RI, Nasr C, Bischoff L, Busaidy NL, Byrd D, Callender G, et al. NCCN Guidelines Insights: Thyroid Carcinoma, Version 2.2018[J]. J Natl Compr Canc Netw. 2018;16(12):1429-40.

11. Expert Panel On Radiation Oncology-Bone M, Lutz ST, Lo SS, Chang EL, Galanopoulos N, Howell DD, et al. ACR Appropriateness Criteria(R) non-spine bone metastases[J] J Palliat Med. 2012,15(5):521-6.

12. Expert Panel on Radiation Oncology-Bone M, Lo SS, Lutz ST, Chang EL, Galanopoulos N, Howell DD, et al. ACR Appropriateness Criteria (R) spinal bone metastases[J] J Palliat Med. 2013,16(1):9-19.

13. Hirshberg A, Leibovich P, Buchner A. Metastatic tumors to the jawbones: analysis of 390 cases[J]. J Oral Pathol Med. 1994;23(8):337-41. 
14. Mai KT, Khanna P, Yazdi HM, Perkins DG, Veinot JP, Thomas J, et al. Differentiated thyroid carcinomas with vascular invasion: a comparative study of follicular, Hurthle cell and papillary thyroid carcinoma[J]. Pathology. 2002;34(3):239-44.

15. van der Waal RI, Buter J, van der Waal I. Oral metastases: report of 24 cases[J]. Br J Oral Maxillofac Surg. 2003;41(1):3-6.

16. Glaser C, Lang S, Pruckmayer M, Millesi W, Rasse M, Marosi C, et al. Clinical manifestations and diagnostic approach to metastatic cancer of the mandible[J]. Int J Oral Maxillofac Surg. 1997;26(5):365-8.

17. Ismail SB, Abraham MT, Zaini ZB, Yaacob HB, Zain RB. Metastatic follicular thyroid carcinoma to the mandible: a case report[J]. Cases J. $2009 ; 2: 6533$.

18. Tamiolakis D, Tsamis I, Thomaidis V, Lambropoulou M, Alexiadis G, Venizelos I, et al. Jaw bone metastases: four cases[J] Acta dermatovenerologica Alpina, Pannonica, et Adriatica. 2007,16(1):21-5.

19. Anil S, Lal PM, Gill DS, Beena VT. Metastasis of thyroid carcinoma to the mandible. Case report[J]. Australian dental journal. 1999;44(1):56-7.

20. Vural E, Hanna E. Metastatic follicular thyroid carcinoma to the mandible: a case report and review of the literature[J]. Am J Otolaryngol. 1998;19(3):198-202.

21. Haugen BR, Alexander EK, Bible KC, Doherty GM, Mandel SJ, Nikiforov YE, et al. 2015 American Thyroid Association Management Guidelines for Adult Patients with Thyroid Nodules and Differentiated Thyroid Cancer: The American Thyroid Association Guidelines Task Force on Thyroid Nodules and Differentiated Thyroid. Cancer[J] Thyroid. 2016;26(1):1-133.

22. Mandell RB, Mandell LZ, Link CJ. Jr. Radioisotope concentrator gene therapy using the sodium/iodide symporter gene[J]. Cancer research. 1999;59(3):661-8.

23. Fozzatti L, Velez ML, Lucero AM, Nicola JP, Mascanfroni ID, Maccio DR, et al. Endogenous thyrocyte-produced nitric oxide inhibits iodide uptake and thyroid-specific gene expression in FRTL-5 thyroid cells[J]. J Endocrinol. 2007;192(3):627-37.

24. Bizhanova A, Kopp P. Minireview. The sodium-iodide symporter NIS and pendrin in iodide homeostasis of the thyroid[J]. Endocrinology. 2009;150(3):1084-90.

25. Min JJ, Chung JK, Lee YJ, Jeong JM, Lee DS, Jang JJ, et al. Relationship between expression of the sodium/iodide symporter and 131/ uptake in recurrent lesions of differentiated thyroid carcinoma[J]. Eur J Nucl Med. 2001;28(5):639-45.

26. Lin JD, Chao TC. Follicular thyroid carcinoma: From diagnosis to treatment[J]. Endocr J. 2006;53(4):441-8.

27. Hirshberg A, Buchner A. Metastatic tumours to the oral region. An overview[J]. Eur J Cancer B Oral Oncol. 1995;31B(6):355-60.

28. Osguthorpe JD, Bratton JR. Occult thyroid carcinoma appearing as a single mandibular metastasis[J] Otolaryngology-head and neck surgery: official journal of American Academy of Otolaryngology-Head and Neck Surgery. 1982,90(5):674-5.

29. Markitziu A, Fisher D, Marmary Y. Thyroid papillary carcinoma presenting as jaw and parotid gland metastases[J]. Int J Oral Maxillofac Surg. 1986;15(5):648-53.

30. Hanahan D, Weinberg RA. The hallmarks of cancer[J]. Cell. 2000;100(1):57-70.

31. Zachariades N, Koumoura F, Vairaktaris E, Mezitis M. Metastatic tumors to the jaws: a report of seven cases[J]. Journal of oral maxillofacial surgery: official journal of the American Association of Oral Maxillofacial Surgeons. 1989;47(9):991-6.

32. Kricun ME. Red-yellow marrow conversion: its effect on the location of some solitary bone lesions[J]. Skeletal Radiol. 1985;14(1):10-9.

33. Pruckmayer M, Glaser C, Marosi C, Leitha T. Mandibular pain as the leading clinical symptom for metastatic disease: nine cases and review of the literature[J]. Annals of oncology: official journal of the European Society for Medical Oncology. 1998;9(5):559-64.

34. Kahn MA, McCord PT. Metastatic thyroid carcinoma of the mandible: case report[J]. Journal of oral maxillofacial surgery: official journal of the American Association of Oral Maxillofacial Surgeons. 1989;47(12):1314-6.

35. Liu L, Venkataraman G, Salhadar A. Follicular variant of papillary thyroid carcinoma with unusual late metastasis to the mandible and the scapula[J]. Pathology international. 2007;57(5):296-8.

36. K CL. R, M V. Mandibular metastasis of thyroid carcinoma: a case report[J]. Journal of clinical diagnostic research: JCDR. 2014;8(7):Zd15-6.

37. Nawale KK, Vyas M, Kane S, Patil A. Metastatic tumors in the jaw bones: A retrospective clinicopathological study of 12 cases at Tertiary Cancer Center[J]. Journal of oral maxillofacial pathology: JOMFP. 2016;20(2):252-5.

38. Slim I, Mhiri A, Meddeb I, Goucha A, Gritli S, Ben Slimene MF. Malar bone metastasis revealing a papillary thyroid carcinoma[J] Case reports in otolaryngology. 2012,2012:795686.

39. Algahtani M, Alqudah M, Alshehri S, Binahmed A, Sandor GK. Pathologic fracture of the mandible caused by metastatic follicular thyroid carcinoma[J]. J Can Dent Assoc. 2009;75(6):457-60.

40. Nikitakis NG, Polymeri A, Polymeris A, Sklavounou A. Metastatic papillary thyroid carcinoma to the maxilla: case report and literature review[J]. Head neck pathology. 2012;6(2):216-23.

41. Agarwal A, Mishra SK, Jain M. Follicular thyroid carcinoma with metastasis to the mandible[J]. J Indian Med Assoc. 1998;96(11):354-5.

42. Bhadage CJ, Vaishampayan S, Umarji H. Mandibular metastasis in a patient with follicular carcinoma of thyroid[J]. Contemp Clin Dent. 2012;3(2):212-4.

43. Kumar CS, Shanmugam D, Venkatapathy R, Munshi MA. Metastatic follicular carcinoma of thyroid in maxilla[J]. Dent Res J (Isfahan). 2013;10(6):817-9.

44. Hefer T, Manor R, Zvi Joachims H, Groisman GM, Peled M, Gov-Ari E, et al. Metastatic follicular thyroid carcinoma to the maxilla[J]. J Laryngol Otol. 1998;112(1):69-72.

45. Fatahzadeh M, Subramanian G, Singer SR. Metstatic papillary thyroid carcinoma to maxilla: a rare case[J] Quintessence international (Berlin, Germany: 1985). 2015,46(5):431-5.

46. Antunes AA, Antunes AP. Gnathic bone metastasis: a retrospective study of 10 cases[J]. Braz J Otorhinolaryngol. 2008;74(4):561-5.

Page 10/14 
47. Zandi M, Jafari M, Isapour M, Jafari AA. Mandibular metastasis in a patient with undiscovered synchronous thyroid and prostate cancer: A diagnostic dilemma[J]. Journal of oral maxillofacial pathology: JOMFP. 2014;18(3):449-52.

48. Bingol UA, Cinar C, Cetinkale O. Mandibular metastases of papillary thyroid carcinoma treated by hemimandibulectomy and costochondral rib graft[J] Plastic and reconstructive surgery Global open. 2015,3(2):e305.

49. Colella G, Capone R, Cappabianca S. Mandibular metastasis from papillary thyroid carcinoma-a case report[J]. Tumori. 2003;89(4):452-4.

50. Draper BW, Precious DS, Priddy RW, Byrd DL. Clinicopathological conference. Case 29, part 2. Follicular thyroid carcinoma metastatic to the mandible[J] Journal of oral surgery (American Dental Association: 1965). 1979,37(10):736-9.

51. Erdag T, Bilgen C, Ceryan K. Metastatic thyroid carcinoma of the mandibule[J]. Rev Laryngol Otol Rhinol (Bord). 1999;120(1):31-4.

52. Essakalli L, Jazouli N, Kzadri M. [Mandibular metastasis disclosing a papillary carcinoma of the thyroid gland][J]. Rev Stomatol Chir Maxillofac. 1994;95(5):351-4.

53. Germain MA, Marandas P, Leridant AM, Domenge C, Julieron M, Schlumberger M, et al. [Isolated mandibular metastasis of cancer of the thyroid. Mandibulectomy and reconstruction using a free vascularized peroneal graft][J]. Rev Stomatol Chir Maxillofac. 1998;98(6):371-4.

54. Kumar RV, Chakravarthy C, Sekhar GR, Devireddy SK, Kumaravelu C, Kare A. Metastatic thyroid carcinoma presenting as hypervascular lesion of the mandible: a case report and review of literature[J]. Journal of oral maxillofacial surgery: official journal of the American Association of Oral Maxillofacial Surgeons. 2010;68(10):2613-6.

55. Vishveshwaraiah PM, Mukunda A, Laxminarayana KK, Kasim K. Metastatic follicular thyroid carcinoma to the body of the mandible mimicking an odontogenic tumor[J]. J Cancer Res Ther. 2013;9(2):320-3.

56. Vazifeh Mostaan L, Irani S, Rajati M, Memar B. Mandibular metastasis from follicular thyroid carcinoma: a rare case after twelve years[J]. Arch Iran Med. 2013;16(9):557-9.

57. Tovi F, Leiberman A, Hirsch M. Uncommon clinical manifestations in a case of thyroid carcinoma[J]. Head Neck Surg. 1984;6(5):974-7.

58. Pasupula AP, Dorankula SP, Thokala MR, Kumar MP. Metastatic follicular thyroid carcinoma to the mandible[J]. Indian journal of dental research: official publication of Indian Society for Dental Research. 2012;23(6):843.

59. Ostrosky A, Mareso EA, Klurfan FJ, Gonzalez MJ. Mandibular metastasis of follicular thyroid carcinoma. Case report[J] Med Oral. 2003;8(3):224-7.

60. Meyer I, Shklar G. Malignant Tumors Metastatic To Mouth and Jaws[J]. Oral Surg Oral Med Oral Pathol. 1965;20:350-62.

61. Nishikawa H, Nakashiro K, Sumida T, Sugita A, Hamakawa H. Mandibular osteoblastic metastasis of poorly differentiated carcinoma of the thyroid gland[J]. Int J Oral Maxillofac Surg. 2010;39(3):301-4.

62. McDaniel RK, Luna MA, Stimson PG. Metastatic tumors in the jaws[J]. Oral Surg Oral Med Oral Pathol. 1971;31(3):380-6.

\section{Figures}
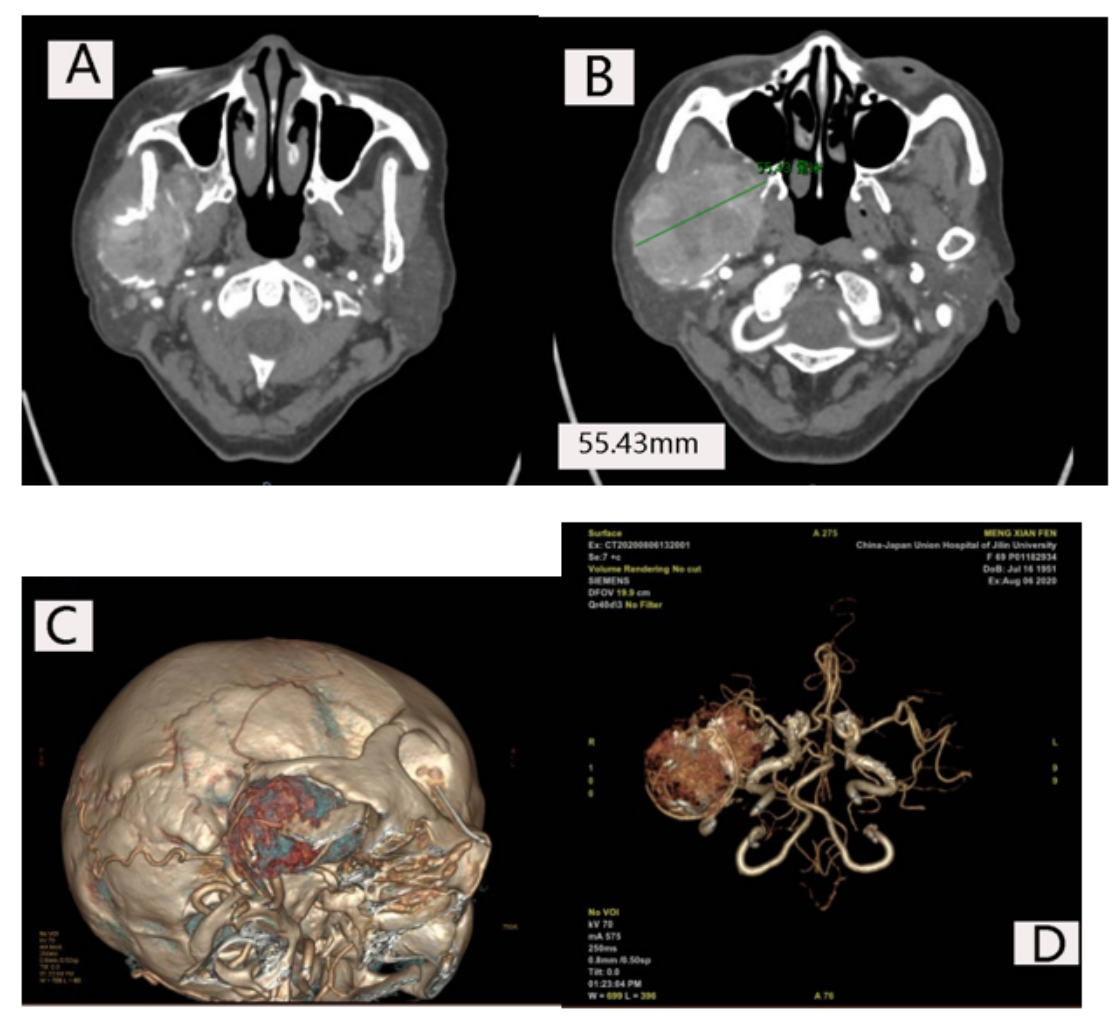

Figure 1 
Computed tomography angiography of the carotid showed marked bony destruction in the involved mandible (A), the size of the space-occupying lesions at the right parotid region (B) and angiography (C and D).
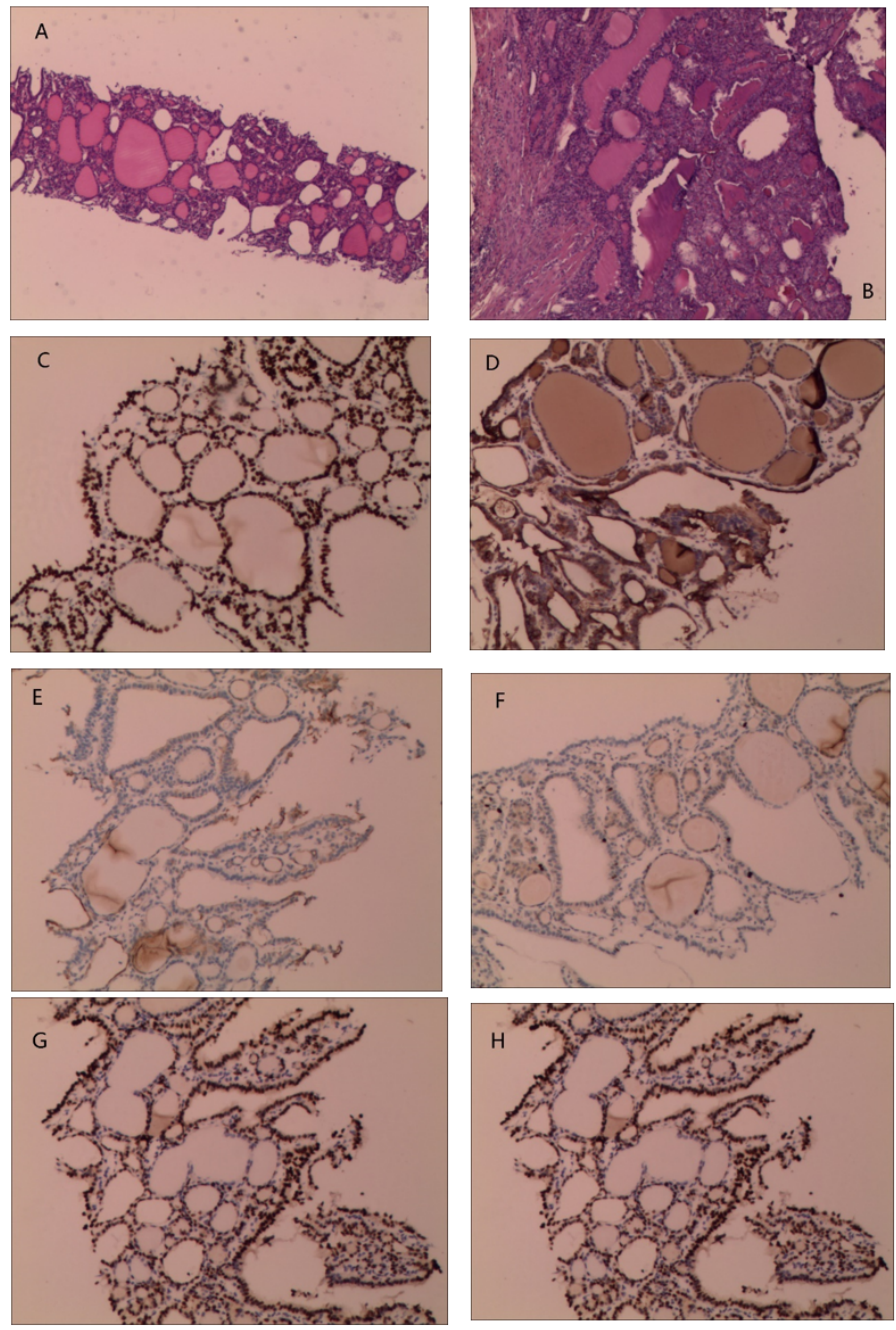

\section{Figure 2}

Hematoxylin and eosin staining of the biopsy at 40X (A) and 100X (B); images show infiltrating tumor cells. Immunohistochemical analysis of nodule revealed TTF1 (C) and thyroglobulin (D) positivity; CK7 (E), PAX-8 (G) and galectin-3 (H) staining showed positivity; and the Ki67 index was $5 \%$ (F). 

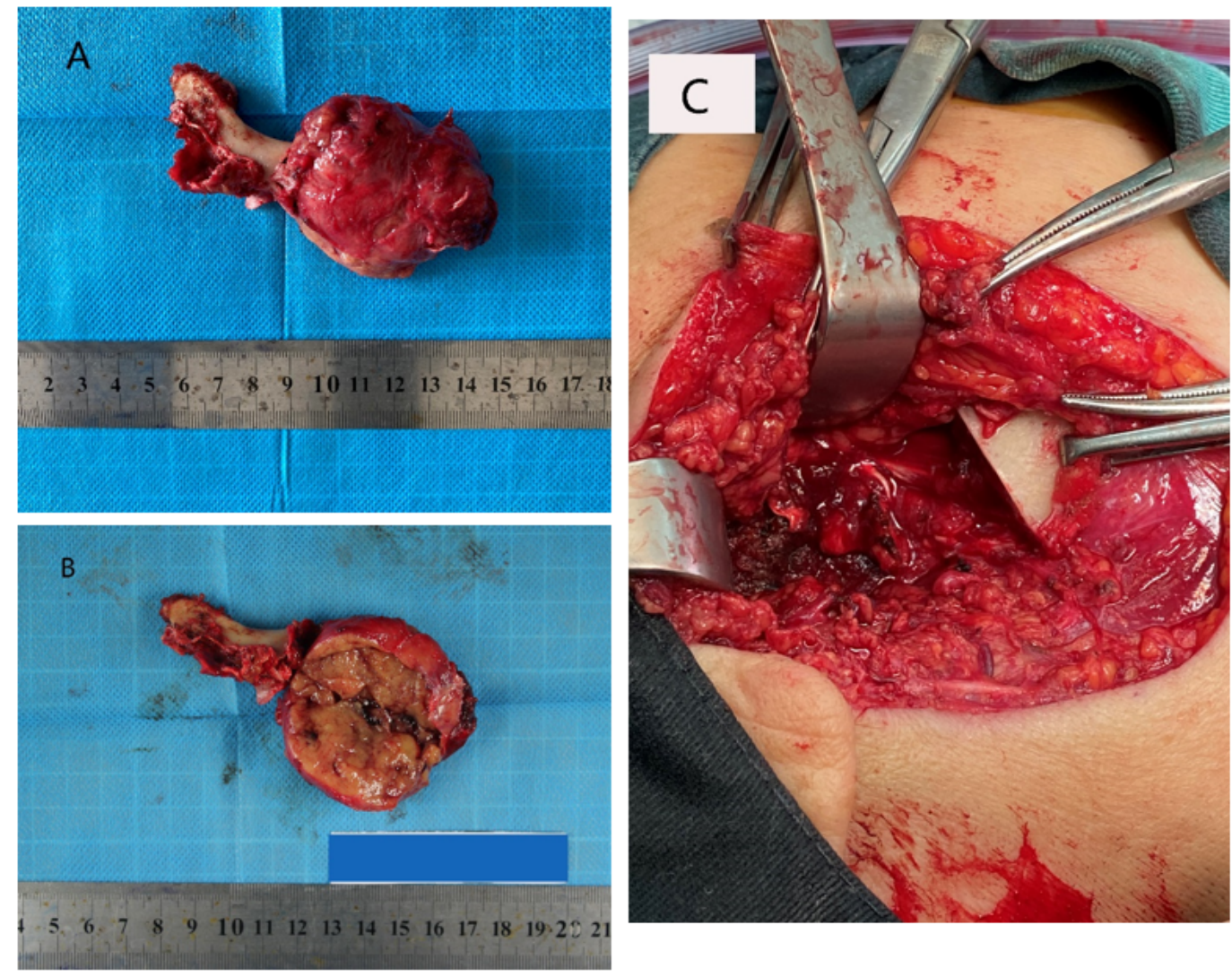

\section{Figure 3}

Pathology image (A) shows gross macroscopic pictures of the excised specimen; (B) the tissue specimen was cut lengthwise to reveal its interior; (C) the operative cavity after removal of the tumor. 

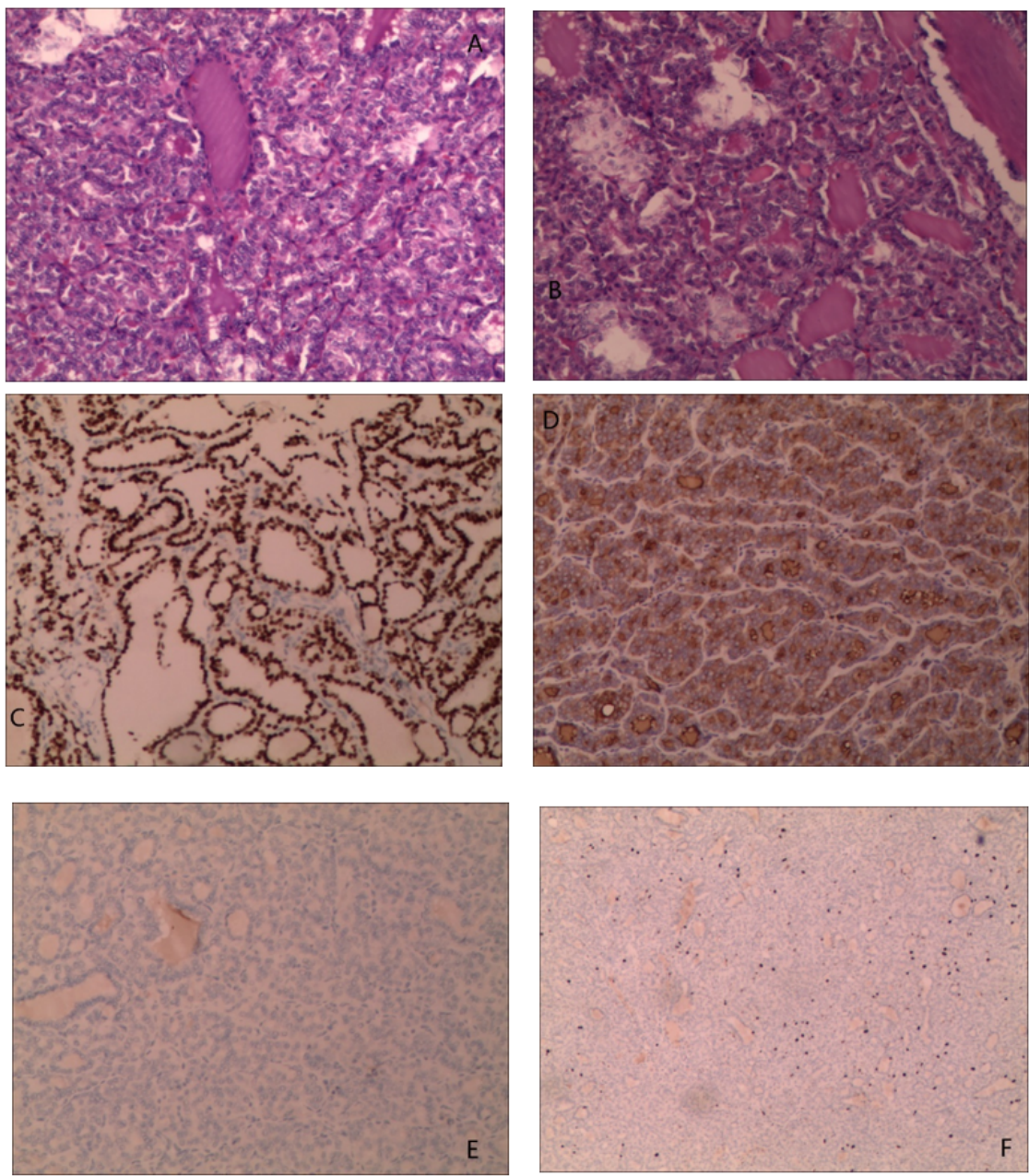

\section{Figure 4}

Hematoxylin and eosin staining at 40X (A) and 100X (B) depicting tumor cells infiltrating and destroying the mandibular bone after the operation. Immunohistochemical analysis of the nodule revealed TTF1 (C) and thyroglobulin (D) positivity; CK-19 (E) showed positivity; and the Ki67 index was 5\% (F). 